\title{
La vivencia del rechazo en homosexuales universitarios de la Ciudad de México y situaciones de riesgo para VIH/SIDA
}

\author{
José Arturo Granados-Cosme, M en MS,(1) César Torres-Cruz, L en CS, ${ }^{(1)}$ \\ Guadalupe Delgado-Sánchez, L en N.(2)
}

\section{Granados-Cosme JA,Torres-Cruz C, Delgado-Sánchez G. La vivencia del rechazo en homosexuales universitarios de la Ciudad de México y situaciones de riesgo para $\mathrm{VIH} /$ sida. Salud Publica Mex 2009;5 I:482-488.}

\begin{abstract}
Resumen
Objetivo. Describir la relación que establece un grupo de varones homosexuales entre la percepción del rechazo social a su homosexualidad, sus prácticas sexuales y el riesgo de $\mathrm{VIH} /$ sida. Material y métodos. Se aplicaron entrevistas a profundidad a homosexuales de una universidad de la Ciudad de México reclutados mediante la técnica de "bola de nieve". Las entrevistas se grabaron y procesaron en el programa Atlas. ti y el análisis del discurso se realizó con el método denominado "teoría fundamentada". Resultados. La experiencia de la homofobia que manifestaron los entrevistados generó sufrimiento psíquico, incluidos tristeza, miedo y conducta suicida, asociado con situaciones de riesgo para $\mathrm{VIH} /$ sida: escasa comunicación, baja negociación para usar el condón y prácticas de riesgo como la penetración anal. Conclusiones. La homofobia, traducida en discriminación, incrementa la vulnerabilidad a la transmisión sexual del VlH.
\end{abstract}

Palabras clave: homosexualidad; homofobia; $\mathrm{VIH} /$ sida; investigación cualitativa; México
Granados-Cosme JA,Torres-Cruz C, Delgado-Sánchez G.

The experience of rejection in homosexual

university students from Mexico City

and risk situations for HIVIAIDS.

Salud Publica Mex 2009;5 I:482-488.

\section{Abstract}

Objective.To describe the relationship established by a male homosexual group between the perception of social rejection towards their homosexuality, sexual practices and risk of HIVIAIDS. Material and Methods. In-depth interviews were administered to homosexual men at a Mexico City university by means of a snowball technique. The interviews were recorded and processed with Atlas.ti software and the analysis of the discourse was made using the Grounded Theory method. Results. The experience of homophobia as expressed by the interviewees resulted in psychological suffering, including sadness, fear, loneliness and suicidal behavior associated with risk situations for HIVIAIDS. Conclusions. Homophobia, translated into discrimination, contributes to an increased vulnerability to the sexual transmission of HIV.

Key words: homosexuality; homophobia; HIVIAIDS; qualitative research; Mexico

(I) Universidad Autónoma Metropolitana. México DF, México.

(2) Instituto Nacional de Salud Pública. Cuernavaca, Morelos, México.

Fecha de recibido: 3 de septiembre de 2008 - Fecha de aceptado: 23 de julio de 2009 Solicitud de sobretiros: Mtro. José Arturo Granados-Cosme. Universidad Autónoma Metropolitana. Calz. del Hueso I I00, col.Villa Quietud. 04960 Coyoacán, México, DF

Correo electrónico: jcosme@correo.xoc.uam.mx 
Es el caso de la sociedad mexicana, la cultura sobre sexualidad está conformada a partir de una visión polarizada de los géneros ${ }^{1} \mathrm{y}$ matizada por un gran conservadurismo, ${ }^{2}$ en virtud del cual el heterosexismo se ha impuesto como la base de la normatividad sexual. Como mecanismos para hacer prevalecer este rasgo se han identificado el machismo ${ }^{3}$ y la homofobia ${ }^{4}$ que, pese a ciertos cambios, aún predominan. Al igual que en otros contextos culturales, la homofobia forma parte en México de los ejes sobre los que se construye el modelo dominante de masculinidad. ${ }^{5,6}$

La homofobia se ha relacionado con la vulnerabilidad de los varones homosexuales a la transmisión del $\mathrm{VIH} /$ sida, ya que ocasiona en esa población mayores tasas globales de trastornos mentales en comparación con los heterosexuales; ${ }^{7}$ en particular se ha reconocido una mayor prevalencia de trastornos depresivos, $, 8,9$ ansiedad ${ }^{8}$ ataques de pánico y estrés psicológico, ${ }^{7,9-15}$ trastornos que a su vez se han asociado con una mayor presencia de prácticas sexuales de riesgo en este sector. ${ }^{16-19}$

En el caso de México, las prevalencias más elevadas de $\mathrm{VIH} /$ sida aún se concentran en varones en edad productiva y la vía de transmisión más frecuente es la homosexual, ${ }^{20}$ de ahí la importancia de profundizar en los procesos sociales que suponen un mayor riesgo de $\mathrm{VIH} /$ sida en los varones homosexuales. ${ }^{19,21-24}$

Una forma de captar los componentes culturales de una sociedad consiste en documentar la experiencia de los sujetos. En el caso de la cultura sobre la sexualidad, ésta es susceptible de recuperarse mediante las experiencias que las personas han tenido en relación con la normatividad sexual que, como ya se mencionó, es heterocentrista y basada en un modelo masculino inherentemente homofóbico. Esta finalidad requiere un abordaje cualitativo toda vez que es el más recomendable para identificar los significados y prácticas que constituyen la cultura.

Con el objetivo de describir la relación que un grupo de varones homosexuales establece con su percepción de la homofobia, el sufrimiento psíquico que le ocasiona y la configuración de situaciones de riesgo para la transmisión del VIH-sida, se diseñó un estudio cuya metodología se orientó a recuperar la experiencia como proceso reflexivo que permite captar la incorporación de significados y prácticas predominantes sobre la sexualidad, así como la incorporación de la homofobia en la conformación del sistema identitario de los homosexuales, su expresión como sufrimiento psíquico ${ }^{10,25} \mathrm{y}$ el riesgo de contraer VIH-sida. ${ }^{26}$

\section{Material y métodos}

Se realizó un estudio cualitativo, descriptivo, correlacional, no experimental y transversal, revisado y aprobado por el consejo encargado de dictaminar los proyectos de investigación en la institución de adscripción de los autores. Se aplicaron entrevistas a profundidad a 19 jóvenes varones homosexuales de una universidad de la Ciudad de México, quienes se reclutaron mediante la técnica de la "bola de nieve"; el primer contacto se estableció a través de una organización estudiantil que promueve información sobre sexualidad; las entrevistas se efectuaron entre enero y octubre del año 2006, la duración promedio fue de dos horas y media, y las aplicaron y transcribieron los propios autores. La captación de los entrevistados se limitó cuando se encontraron suficientes regularidades discursivas, es decir, una vez que se "saturó" el discurso. Antes de cada entrevista se entregó información sobre las finalidades del estudio y se garantizó el resguardo de la identidad de los entrevistados, para lo cual en la transcripción de las entrevistas se utiliza un nombre ficticio; también se puso a su disposición un reporte con los resultados del estudio. En todos los casos se entregó un directorio de centros de atención psicológica en el cual los informantes podían recibir atención especializada en caso necesario y, de manera adicional, se contó con la colaboración de profesionales de la salud mental para derivar a los informantes que manifestaran sufrimiento psíquico considerable, especialmente a aquellos que en el momento de la entrevista mostraran conductas suicidas.

La guía de la entrevista incluyó: a) experiencia de la homofobia interpersonal y la observada hacia otros homosexuales en los entornos de socialización primaria y secundaria; y b) sufrimiento psíquico causado por la homofobia y riesgos potenciales y reales para contraer el VIH/sida (figura 1). Las entrevistas se grabaron, transcribieron y procesaron en el programa Atlas.ti; el análisis de datos se llevó a cabo bajo la perspectiva de la teoría fundamentada del análisis del discurso. ${ }^{27}$, ${ }^{28}$ Con este término se hace referencia sólo al método de interpretación de datos que sus autores llamaron "teoría", no al sentido estricto del término que remite a la formulación de hipótesis basadas en un cuerpo de conocimientos específicos.

\section{Resultados}

Se entrevistó a 19 jóvenes varones homosexuales cuya edad osciló entre los 20 y los 28 años de edad; la edad 
media fue de 22 años, todos eran estudiantes de licenciatura de las áreas sociales, humanidades, artes y diseño, así como biología y ciencias de la salud; y todos se identificaron a sí mismos como homosexuales.

\section{Experiencia de la homofobia y salud mental}

Respecto de la experiencia de la homofobia, los discursos de los informantes se analizaron según lo muestra la figura 2. Los entrevistados tuvieron conocimiento de

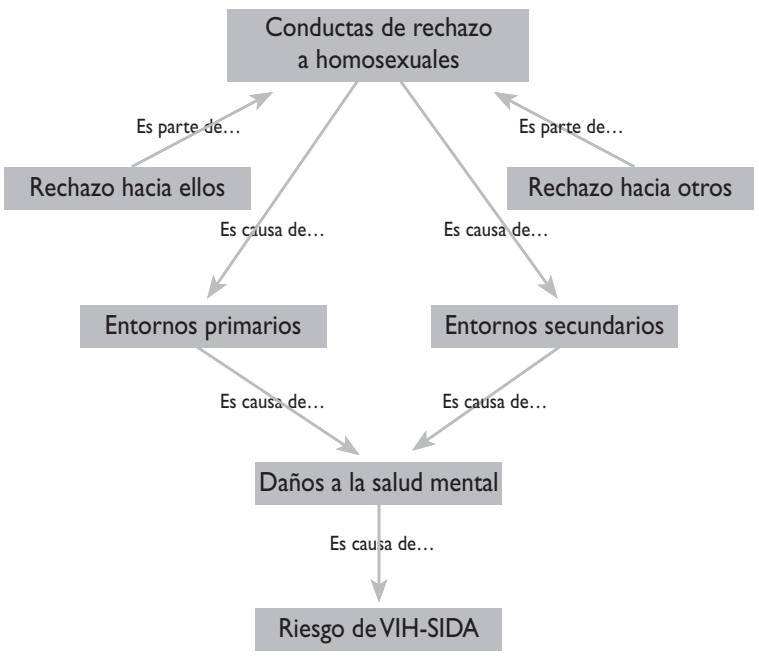

Figura I. HOMOFObia Y SALUd

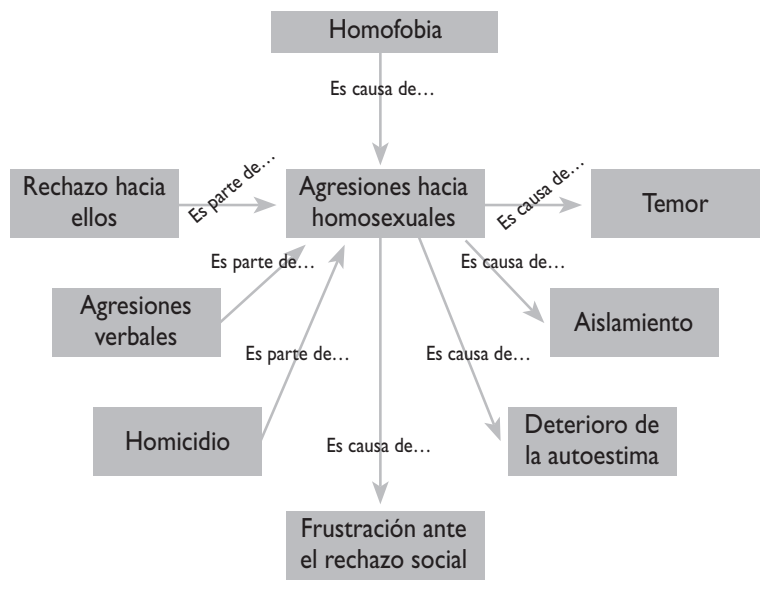

Figura 2. HOMOFObia Y DAÑos A LA SALUd MENTAL conductas de rechazo a la homosexualidad en general, hacia sí mismos y hacia otros homosexuales, tanto en sus entornos primarios (familia, hogar) como en los secundarios (escuela, trabajo y espacios públicos), lo cual generó sentimientos de temor y tristeza, reforzó estrategias de evitación a través del ocultamiento de la orientación sexual y provocó aislamiento. La categoría homofobia se expresó por medio de códigos verbales cuyos significados denotan devaluación ('puto', 'joto', 'mariquita', 'niña'), violencia física ('agredían', 'pegaban') y exclusión ('enfermedad'), cuyo significado es la devaluación ('asco', 'compadezco', 'pobrecitos').

íbamos saliendo del metro ( ) íbamos pasando ( ) un amigo y yo y se nos cruza uno así de: ‘¡ah pinches putos!" [Joaquín].

...Cuando iba en secundaria ( ) había un muchacho que ( ) era muy amanerado. Le decían 'joto', lo insultaban, ¡a él sí lo insultaban y lo agredían! (...) ¡Le pegaban! (...) pero yo no me juntaba con él ( ) Me daba ( ) miedo de que también me fueran a agredir [René].

no supe ni cómo fue, pero cuando se enteraron (en mi casa) me pegaron muy fuerte ( ) mi papá, mi mamá, mis dos hermanas ( ) me dolió mucho que me haya pegado mi papá [Pedro].

Sí, sí mi mamá es más de ( ) eh... lastimosa, sí de: ‘jay pobrecitos los compadezco!' [a los homosexuales] ( ) ella piensa que es como una enfermedad ( ) mi papá es más de rechazo, rayando como en el desprecio y el asco [Alexis].

Las agresiones más referidas por los informantes fueron las verbales, dirigidas tanto a otros homosexuales como a ellos, aunque también señalaron agresiones físicas, abuso sexual y aun homicidio. En estos casos, los códigos discursivos con que se expresó esta homofobia fueron: 'burlarse', 'golpearlos', 'tocar' y 'mataron'. Como puede verse, estas formas de homofobia tuvieron un impacto que se expresó con los códigos verbales de la categoría de sufrimiento psíquico ('miedo', 'dolor', 'vergüenza', 'compasión', 'triste'), con sus respectivos correlatos: temor, deterioro de la autovaloración, aislamiento ('solo', 'desesperado') y frustración por no saber cómo enfrentar el rechazo.

[¿Llegaste a ver ataques en ti o en otros homosexuales?] ambas ( ) me tocó ver cómo atacaban a algunas personas y la agresión hacia mi persona. [¿En dónde?] En la escuela, con compañeros de salón [¿Qué les ha- 
cían?] Pues era golpearlos, era burlarse, decirles que eran niñas [Felipe].

mis hermanos me decían: 'pareces mariquita', 'compórtate como hombrecito' antes de que supieran que yo era gay decían que sería mejor que se murieran [los gays] o cosas así. Que era una vergüenza para los hombres que hubiera homosexuales [Antonio].

[¿Te llegaron a golpear alguna vez?] Ah, sí ( ) Uno de mis compañeros ( ) había muchos compañeros que me empezaban a tocar o quererme tocar, o quererme abrazar ( ) también me querían besar, me querían tocar el trasero... [José].

A él [un gay] lo mataron (...) lo habían matado porque él quería con un muchacho [Patricio].

\section{Situaciones y prácticas de riesgo para VIH-sida}

Para este apartado, los discursos de los informantes se analizaron como se ilustra en la figura 3. Al ejercer su sexualidad, la mayoría de los entrevistados enfrentó situaciones de riesgo para contraer infecciones de transmisión sexual (ITS) o VIH/sida y mencionaron la realización de prácticas sexuales de riesgo. Cabe distinguir entre prácticas de riesgo y situaciones de riesgo; en el primer caso, se hace referencia a relaciones sexuales que implican intercambio de fluidos corporales, que contienen los agentes causales de ITS y VIH-sida en cantidades suficientes para la transmisión de la enfermedad; en cambio, con las situaciones de riesgo se alude a las condiciones contextuales en las cuales ocurren las prácticas sexuales. El rechazo social como condición que prevalece en el contexto determina las posibilidades de los interactuantes para ejercer prácticas sexuales seguras o protegidas. Entre las condiciones que conforman las situaciones en las que se ejerce la sexualidad, adquieren importancia las siguientes: a) el conocimiento de las medidas preventivas (conocer los mecanismos de transmisión de las ITS y el VIH, así como el uso del condón), b) la disponibilidad y acceso a los medios materiales para la prevención (existencia de condones, posibilidad de que las personas los adquieran sin dificultades y habilidades necesarias para su uso correcto), y c) la capacidad para negociar de forma explícita (comunicación interpersonal sobre el tipo de prácticas sexuales que se realizarán). Éstas son condiciones relevantes para el uso efectivo del condón y pueden verse afectadas por diversas circunstancias, como el carácter de marginalidad o legitimidad social en que los individuos ejercen su sexualidad; en el caso de los varones homosexuales, el rechazo social genera

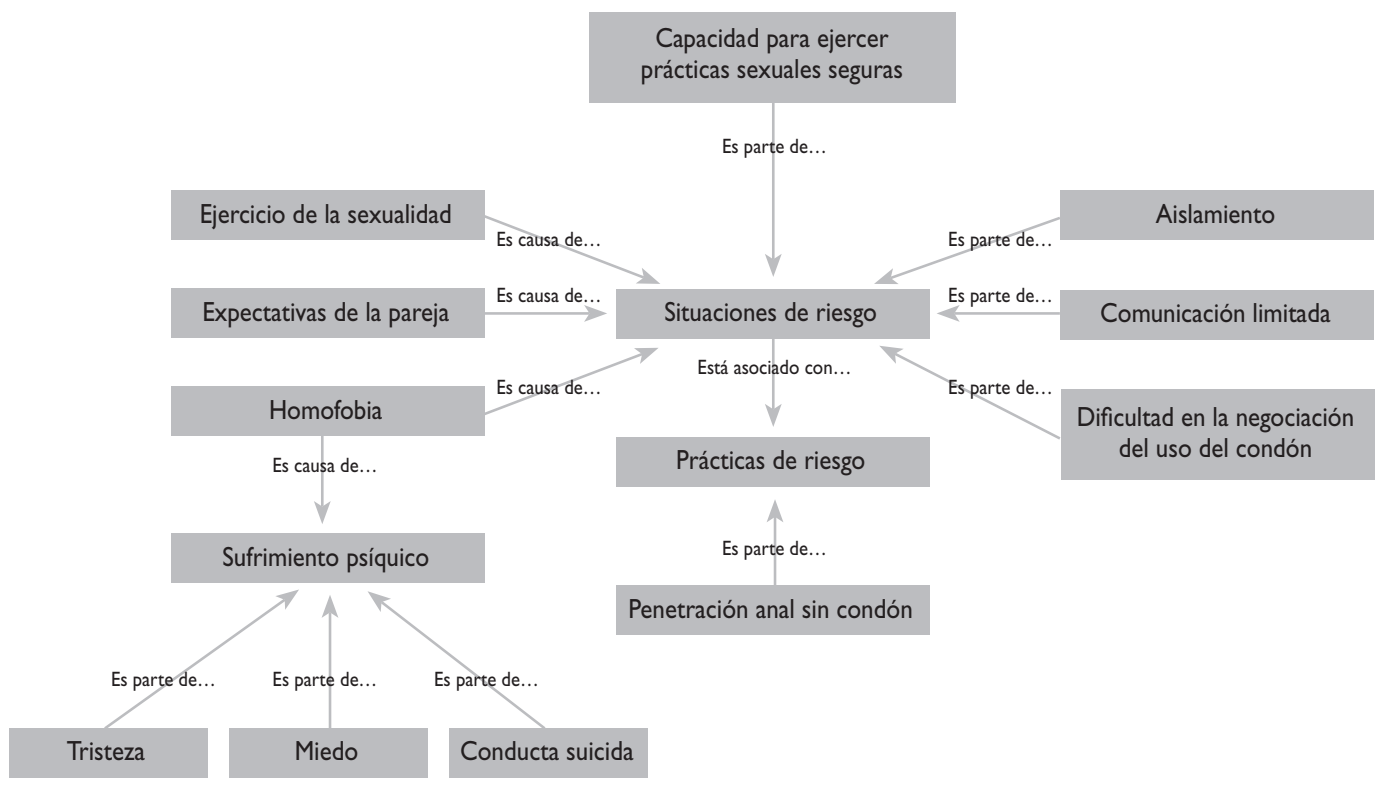

FiguRA 3. INTERACCIÓN ENTRE SUFRIMIENTO PSIQUICO, SITUACIONES DE RIESGO Y PRÁCTICAS DE RIESGO 
anonimato, comunicación limitada y facilita la coerción y otras condiciones.

En su dimensión individual, el rechazo social a la homosexualidad promueve trayectorias individuales marcadas por el aislamiento social, el sufrimiento psíquico y la autovaloración negativa que reducen las capacidades individuales para enfrentar situaciones de riesgo, entre ellas la utilización personal del condón o la exigencia de que la pareja sexual lo use. Los entrevistados narraron diversas situaciones de riesgo potencial y real para la transmisión de ITS y VIH/ sida.

En la categoría de sexualidad se identificaron códigos verbales que denotan situaciones de riesgo y marginalidad ('me fui al cerro'), escasa comunicación y negociación para el uso del condón, sentimientos de coerción ('desconocidos', 'me presionó') y prácticas sexuales de riesgo ('anal', 'sin condón').

entonces, en el metro, ahí empecé a conocer más gays ( ) en vez de irme a la escuela, me iba al metro, a buscar ( ) teníamos relaciones en los baños ( ) con penetración, oral y anal [y ¿usabas condón?] No ( ) rara era la ocasión cuando lo llegaba a usar ( ) seguía siendo con chavos desconocidos [Luis].

Una característica de los encuentros sexuales con prácticas de riesgo es la mezcla de sentimientos de tristeza y temor al buscar vínculos eróticos afectivos; también se mencionan sentimientos de soledad e insatisfacción después de encuentros ocasionales, situación que acentuó la frustración y propició nuevas búsquedas en condiciones similares de riesgo. La asociación entre sufrimiento psíquico, situaciones y prácticas sexuales de riesgo muestra la influencia de la homofobia sobre el mayor riesgo de transmitir el VIH-sida en grupos estigmatizados, al combinarse códigos discursivos como: 'contactos sexuales', 'solo' o 'desesperado'; otras asociaciones discursivas son: 'triste', 'me presionó', 'miedo' y 'no usamos condón'.

[tener contactos sexuales] ha sido cada viernes ( ) ha habido veces que en un viernes es con más de una persona ( ) [pero] me empecé a sentir demasiado solo y desesperado [Ernesto].

Cuando estaba triste me iba al cerro de la Estrella ( ) él dijo que se sentía muy excitado y se bajó su pantalón y me mostró su pene, me dio miedo, pero él me presionó para que terminara y no usamos condón porque me dio miedo y ya quería terminar [Alejandro].

Otras situaciones de riesgo son atribuibles a las idealizaciones sobre la pareja; en estos casos se encuen- tran similitudes con la orientación heterosexual: exclusividad, fidelidad y relación definitiva. Los entrevistados mencionaron que esperaban a 'la pareja definitiva' con la cual establecerían una relación para 'siempre'; estos dos atributos conformaron situaciones de riesgo al omitir la posibilidad de la infidelidad o el cambio de sentimientos propios o de la pareja; los informantes mencionaron que cuando encontraran a su pareja definitiva prescindirían del uso del condón ('no usamos', 'no le gusta'). Los informantes elaboraron argumentos para justificar estas previsiones; las idealizaciones de la pareja tuvieron por contenido códigos verbales: 'lo conozco', 'confío' o 'confianza', 'no me preocupo', pero al indagar sobre el contenido que le atribuyen a estos códigos no consiguen definirlo con claridad y los justifican con códigos discursivos, como 'amor'.

No [usamos condón], es que lo conozco muy ( ) lo que pasa es que fue con mucho amor, mucho cariño y además conozco su historia sexual de arriba abajo ( ) bueno, según lo que él me dice ( ) yo confío mucho en él ( ) entonces, pues no me preocupo demasiado [Andrés].

[¿Has tenido una pareja estable?] A los veinte años () duramos ocho años. [¿Y con él usabas condón?] No, a él no le gustaba el condón. [Durante ese tiempo, ¿llegaste a tener otras parejas ocasionales?] Mmm ( ) no, no, él sí tuvo, él sí tuvo, pero yo no [Jorge].

a mí me pasó de que cuando ya mezclé algún sentimiento de amor, a los cinco meses de que empezamos nuestra relación nos hicimos pruebas para ver si teníamos ( ) algo o no, salimos negativos y pues ya, fue cuando decidimos precisamente practicarlo (el sexo) sin protección [Fernando].

\section{Discusión}

Los entrevistados informaron que han sido víctimas de conductas homofóbicas, sobre todo en la infancia y la adolescencia; los espacios en que ocurrieron fueron, en particular, la familia y la escuela, pero también el trabajo; las agresiones recibidas fueron verbales y físicas. Además, tuvieron conocimiento de agresiones perpetradas en otros homosexuales o las presenciaron y, en ese caso, se narraron las más graves, como el homicidio.

Asimismo, los entrevistados asociaron la homofobia padecida con los sentimientos de tristeza y soledad por la valoración negativa de la homosexualidad; temor por la percepción y experiencia de las agresiones; ideación suicida; $y$, menos a menudo, intento de suicidio por la autovaloración negativa como resultado de la internalización de la homofobia. Ante estos síntomas, los en- 
trevistados reaccionaron con conductas de aislamiento social y conducta suicida (ideación e intento).

Los entrevistados indicaron que ejercieron su sexualidad en situaciones de riesgo para VIH-sida, con escasa comunicación y falta de negociación acerca del tipo de prácticas sexuales y el uso del condón. Las situaciones en que sostuvieron con frecuencia relaciones sexuales se caracterizaron por el anonimato, ansiedad con sentimientos de coerción, temor y necesidad de una valoración positiva. Estas condiciones derivaron en prácticas sexuales de riesgo (penetración anal sin condón). Otros elementos de las situaciones de riesgo fueron la idealización de la pareja sexual como una pareja exclusiva, permanente y definitiva en la que pudiera confiarse, con la expectativa de abandonar el uso del condón.

Estos resultados que mostraron los entrevistados permiten caracterizar la asociación de la homofobia con las situaciones de riesgo para contraer la enfermedad; esto contribuye a comprender el papel que juega el rechazo social de la homosexualidad en el mayor riesgo de transmitir el VIH y la mayor prevalencia de sida en varones homosexuales, en sociedades en las que la pandemia muestra un número más elevado de casos transmitidos por relaciones homosexuales.

En esta asociación, la homofobia forma parte del complejo proceso de determinación social de la mayor vulnerabilidad de los varones homosexuales al VIHsida. El contexto social, a través de las relaciones sociales de inclusión y exclusión, distribuye riesgos y daños a la salud de forma desigual; en el caso de los grupos estigmatizados por su conducta, genera mayores daños a la salud mental; esta inequidad representa un estado intermedio que implica a su vez mayores riesgos para otras enfermedades, además del VIH-sida. Los hallazgos del presente estudio coinciden con otros reportes que indican que los ambientes culturales caracterizados por la intolerancia hacia los homosexuales propician en este sector una serie de problemáticas en salud vinculadas con la experiencia de la opresión y la violencia. ${ }^{10,28}$

Aún es necesario profundizar en la relación entre la homofobia y el VIH-sida y comparar la identificación de la orientación homosexual con el caso de los heterosexuales; esto aportará mayores conocimientos al respecto. Otras vertientes que se abren en el campo de la salud de los homosexuales son las diferencias generacionales, de tal modo que podrían identificarse los cambios sociales en torno de las actitudes hacia la homosexualidad y su efecto en la prevención del VIHsida. El presente trabajo representa una aproximación inicial, pero puede orientar las etapas subsecuentes en esta misma línea.

\section{Referencias}

I. Lamas M. Diferencias de sexo, género y diferencia sexual. Cuicuilco 2000; 7(18): 95-118.

2. González E. La sexualidad prohibida. Intolerancia, sexismo y represión. México: Plaza y Janés, 2002.

3.Aramoni A. Psicoanálisis de la dinámica de un pueblo. México: UNAM, 1961.

4. Lumsden I. Homosexualidad. Sociedad y Estado en México. México:

Solediciones, 1991.

5. Badinter E. XY. La identidad masculina. Madrid:Alianza Editorial, 1992.

6. Loyden H. Los hombres y su fantasma de lo femenino. México:

Universidad Autónoma Metropolitana, 1998.

7. Jorm AF, Korten AE, Rodgers B, Jacomb PA, Christensen H. Sexual orientation and mental health: results from a community survey of young and middle-aged adults. Br J Psychiatry 2002; I80:423-427.

8. Perkins DO, Stern RA, Golden RN, Murphy C, Naftolowitz D, Evans DI. Mood disorders in HIV infection: prevalence and risk factors in a nonepicenter of the AIDS epidemic. Am J Psychiatry 1994; I5I (2):233-236.

9. Cochran SD, Mays VM, Sullivan JG. Prevalence of mental disorders, psychological distress and mental health services use among lesbian gay and bisexual adults in the United States.J Consult Clin Psychol 2003; 7I(I):53-6I.

I0. Erwin K. Interpreting the evidence: competing paradigms and the emergence of lesbian and gay suicide as a "social fact". Int J Health Serv 1993;23(3):437-453.

II. King M, Sminth G, Bartlett A. Treatments of homosexuality in Britain since the 1950s -an oral history: the experience of professionals. BMJ 2004; 8 (7437): 429.

12. Balsam KF, Huang B, Fieland KC, Simoni JM,Walters KL. Culture, trauma, and wellness: a comparison of heterosexual and lesbian, gay bisexual, and two-spirit native Americans. Cult Div Ethn Min Psychol 2004; 10(3):278-30I.

13. Lackner JB, Joseph JG, Ostrow DG, Kessler RC, Eshleman S, Wortmar $\mathrm{CB}$, et al. A longitudinal study of psychological distress in a cohort of gay men. Effects of social support and coping strategies.J Nerv Ment Dis 1993; 18I(I):4-12.

14. Garland AF, Zigler E.Adolescent suicide prevention. Current research and social policy implications. Am Psychol 1993; 48(2): 169-182.

15. Lock J, Steiner H. Gay, lesbian, and bisexual youth risks for emotional, physical, and social problems: results from a community-based survey.J Am Acad Child Adolesc Psych 1999; 38(3): 297-304.

16. Wilson PA, Yoshikawa H. Experiences of and responses to social discrimination among Asian and Pacific Islander gay men: their relationship to HIV risk.AIDS Educ Prev 2004; 16(I):68-83.

17. Beck A, McNally I, Petrak J. Psychosocial predictors of HIV/STI risk behaviours in a sample of homosexual men. Sex Transm Infect 2003; 79 (2): 142-146.

18. Kelly JA, St Lawrence JS, Brasfield TL. Predictors of vulnerability to AIDS risk behavior relapse.J Consult Clin Psychol 1991;59(I): I63-166. 19. Rotheram-Borus MJ, Rosario M, Reid H, Koopman C. Predicting patterns of sexual acts among homosexual and bisexual youths. Am J Psychiatry 1995; 152(4):588-595.

20. Secretaría de Salud. Anuario estadístico 2004. México: Secretaría de Salud, 2004.

2I. Preston DB, D'Augelli AR, Kassab CD, Cain RE, Schulze FW, Starks, $M t$. The influence of stigma on the sexual risk behavior of rural men who have sex with men. AIDS Educ Prev 2004; 16(4):291-303.

22. Delor F, Hubert M. Un ré-examen du concept de «vulnérabilité» pour la recherche et la prévention du VIH/SIDA. Bruselas: Centre d'études sociologiques-Facultés universitaires Saint-Louis, 2003. 
23. Skegg K, Nada-Raja S, Dickson N, Paul C, Williams S. Sexual orientation and self-harm in men and women. Am J Psychiatry 2003; I60(3):54I-546. 24. Huebner DM, Rebchook GM, Kegels SM. Experiences of harassment, discrimination and physical violence among young gay and bisexual men. Am J Public Health 2004; 94(7): 1200- 1203.

25. Meyer IH. Prejudice, social stress, and mental health in lesbian, gay, and bisexual populations: conceptual issues and research evidence. Psychol Bull 2003; 129(5):674-697.
26. Díaz R, Ayala G. Social discrimination and health. The case of latino gay men and HIV risk. Estados Unidos: The policy Institute of the Nacional Gay \& Lesbian Task Force, 200I.

27. Glaser BG, Strauss AL. The discovery of grounded theory. Strategies for qualitative research. London:Weindwnfeld \& Nicolson, 1967.

28. Hamel RE. Language, discourse and cultural models: three levels of language shift and maintenance. Soutw J Ling 1998; I5(I-2): 63-88. 